\title{
Behavior of Manets with Evolutionary Algorithms
}

\author{
Debnath Bhattacharyya ${ }^{1}$, N.Thirupathi Rao ${ }^{2}$ \\ ${ }^{1,2}$ Department of Computer Science \& Engineering, Vignan's Institute of Information \\ Technology (A), Visakhapatnam, AP, India \\ 1'debnathb@gmail.com, ${ }^{2}$ nakkathiru@gmail.com
}

\begin{abstract}
Developmental calculations are metaheuristic calculations that give semi ideal arrangements in a sensible time. They have been connected to numerous enhancement issues in a high number of logical regions. In this paper, we center on the utilization of developmental calculations to take care of improvement issues identified with a kind of complex system like portable multihop specially appointed systems. Since its starting point, portable multihop specially appointed system has advanced causing new kinds of multihop systems to seem, for example, vehicular impromptu systems and postpone tolerant systems, prompting the arrangement of new issues and streamlining issues. In this article, an audit of the primary work introduced for each kind of versatile multihop specially appointed system and additionally exhibit some imaginative thoughts and open difficulties to manage additionally explore in this point.
\end{abstract}

Keywords: MANETs, mobiles, devices, ad hoc networks, security, algorithms, evolutionary models, ABC algorithm, routing, routing protocols.

\section{Introduction}

Portable remote multihop impromptu systems have pulled in the consideration of mainstream researchers for over two decades[1][4]. The fundamental thought of conveying electronic gadgets utilizing a remote multihop way, without the need of a focal framework or a settled foundation, has developed since its source and it is as yet a dynamic focal point of research. The expansion of compact electronic gadgets with remote correspondence abilities like Smartphone's and tablets has made conceivable a universe of remote gadgets that can trade data pervasively. The investigation and examination of portable multihop specially appointed systems have been completed by reproduction investigation utilizing occasion based system test systems. The primary reason is that genuine test beds require a high interest regarding equipment [2][3][5] and, all the more critically, the replication of genuine portable conditions is exceptionally troublesome in a controlled situation like a lab. Subsequently, the fundamental system to assess the execution of multihop impromptu systems has been the utilization of prevalent systems test systems. Numerous execution measurements have been introduced and broke down to enhance the execution of versatile multihop specially appointed systems under various circumstances.

Be that as it may, a few investigations have uncovered that versatile multihop specially appointed systems are unpredictable systems where numerous plan parameters are

Article history:

Received (April 30, 2019), Review Result (June 19, 2019), Accepted (August 3, 2019) 
intercorrelated [6][7] which makes the utilization of logical models to be enhanced from a hypothetical perspective exceptionally troublesome. Indeed, even for the situation that a scientific model is accessible, it might be extremely hard to apply inclination based streamlining components acquire the worldwide most extreme and least qualities. This reality is urging the exploration group to investigate new instruments to advance the execution of versatile multihop impromptu system, for example, by utilizing metaheuristic approaches like developmental calculations.

Transformative calculations are metaheuristic calculations planned to take care of complex enhancement issues. In spite of the fact that they were proposed quite a few years back, their application on genuine streamlining issues requires high computational assets that were not accessible around then. These days, present day PCs and workstations in light of multicore models are equipped for running a great many tasks for each second, making the utilization of transformative calculations a reality in numerous logical regions. They depend on the development of a populace of potential arrangements by the mean of hereditary administrators

like hybrid and transformation. Developmental calculations have been effectively connected to numerous portable multihop enhancement issues, for example, topology administration, broadcasting calculations, steering conventions and portability models, and other related issues. The primary thought is the mix of a transformative calculation into the reproduction structure that executes as the improvement motor to locate the most ideal outline factors for a given advancement issue.

\section{MANETS}

In the current section an attempt had been made to give a brief note on various types of mobile ad hoc networks and their functioning with respect to the various performance measures and their behavior was noted.

\subsection{MANETs}

MANETs speak to the first thought of the multihop worldview [1]. That is, the foundation of an impromptu system shaped by remote gadgets without requiring a focal framework. The first thought was to duplicate the wired correspondences, for example, TCP/UDP and IP based conventions in MANETs. Be that as it may, this unique thought was unrealistic because of the inherent qualities of MANETs as far as versatility and restricted hub's transmission ranges. The two highlights make the topology of MANETs to a great degree alterable. Thus, the conventions initially intended for static system like the Internet can't be effectively adjusted to MANETs [2]. The steering layer has been by a wide margin the most concentrated one[8]. There are some great reviews on directing conventions in the writing[9][8]. The fundamental target is to locate the reasonable correspondence way between a versatile source hub and one or a few portable goal hubs. Moreover, a great directing convention must have the capacity to manage course and connection breakages. The essential component to discover courses is, to be specific, telecom [10].

\subsection{VANETs}

VANETs speak to an advancement of multihop worldview. The thought is to join remote handset into vehicles so they can speak with each other. WAVE innovation in view of IEEE 802.11p is the principle remote innovation imagined to be utilized as a part of VANETs. It is still on the advancement stage however it is relied upon to be incorporated into each business 
vehicle in the blink of an eye. IEEE 802.11p characterizes distinctive levels of organizing the transmitting bundles. One imperative component of WAVE innovation is the thought of Road Site Units (RSUs) that go about as static access focuses, enhancing the worldwide execution of the system. The primary uses of VANETs extend from wellbeing movement applications like crash shirking and street impediment cautioning to activity data and infotainment administrations, for example, amusements and interactive media spilling [1].

\subsection{DTNs}

Another kind of remote multihop versatile system that has been profoundly looked into lately is spoken to by DTNs (defer tolerant systems). They are progressively assembled when cell phones work together to shape correspondence ways while clients are in closeness. They depend on a store-convey and-forward worldview [1], which implies that a hub that needs to hand-off a message starts by putting away it, at that point bears it the system until the point that the transporter experiences the goal or a hub that will probably convey the information near the goal, and afterward at long last advances it. With respect to in DTNs, we can recognize sending calculations and information dispersal calculations [2]. In the previous, the goal is to locate an appropriate sending arrangement to transmit a snippet of data between a source and a goal hub.

Portability in DTNs has pulled in a ton of consideration as of late [3]. The principle thought is to show the versatility of individuals conveying convenient gadgets. These individuals experience each other in controlled situations, for example, logical gatherings and college grounds. Most versatility models for DTNs depend on genuine follows that are gathered through created applications for Smartphone's. Likewise, WiFi coordinate is relied upon to increase facilitate force when it is incorporated into the new ages of PDAs and other versatile gadgets. All the more as of late, DTNs have been utilized for different purposes, for example directed publicizing. One such case is MobiAd [6], an application that gives the client neighborhood commercials in a protection saving way. The promotions are chosen by the telephone from a pool of commercials that are communicated on the nearby versatile base station or got from neighborhood WiFi hotspots.

\section{EVOLUTIONARY ALGORITHMS}

Innovation is developing to a great degree quick giving correspondence abilities to little gadgets. This leads towards profoundly heterogeneous and versatile systems made out of any sort of imparting gadgets, which are not appropriate for existing correspondence systems: selfarranging components are hence required. In nature, there exist numerous natural frameworks that following quite a while of advancement can adapt to the issues we look in these heterogeneous systems: recuperation from disappointment, portability, self-association, solidness, community conduct, et cetera. Analysts are creating nature enlivened calculations take care of complex issues; for instance, they have been broadly connected for organize plan. Next, we give a concise prologue to transformative calculations.

Disconnected methodologies search for the most ideal setup of the calculation that will be later utilized amid the execution. These methodologies for the most part advanced until the point that the ideal is found (on the off chance that it is known) or the nature of the arrangements is assessed through reenactment. On the off chance that the issue fluctuates amid the runtime these disconnected strategies are not appropriate. Online methodologies are utilized amid execution, in these time fluctuating calculations, for adjusting the conduct with the goal that the best following stage is found. They more often than not require serious calculation; accordingly a focal unit may be utilized. Be that as it may, impromptu systems are decentralized 
frameworks; in this way either obliged hubs ready to adapt to exceptionally lightweight Metaheuristic are utilized or disconnected strategies are favored.

A calculation that requires data about the entire framework for a proper activity is said to utilize worldwide learning. This method won't not be constantly appropriate when managing impromptu frameworks in light of the fact that the absence of a focal unit makes the gathering of avant-garde information of the total system troublesome. Just when the information is gotten in advance or the measure of the system is little, we could think about this strategy. In the event that the calculation just uses data got locally, that is, hub's data and hub's neighbors data (utilizing signals or listening in), at that point it is said to utilize nearby information.

All the online strategies we said before managing honey bees and ants [6] utilize worldwide learning. It is conflicting to outline a calculation that is extremely lightweight and runs on the web however require worldwide information. Notwithstanding, we can simply discover a few exemptions where this mix may be doable. For instance in [4], creators utilize a glowworm advancement calculation for expanding the worldwide scope for ideal sensor organization in a self composed manner in the event that hubs are versatile. In this particular issue, having worldwide information already is plausible.

\subsection{CENTRALIZED AND DECENTRALIZED SYSTEMS}

An incorporated framework has a focal unit responsible for advancing or settling on choices for the entire framework. It can utilize either worldwide data or data assembled locally by all hubs, and this data is sent to the focal unit for basic leadership. In the last mentioned, the framework requires huge coordination, expanding, consequently, postponements and overhead. On the off chance that the focal unit comes up short, the entire framework crashes. At the point when hubs locally execute and decide, changing future practices as indicated by the outcomes acquired, it is then viewed as a decentralized framework. It isn't practical to plan an online streamlining process focusing on a decentralized portable impromptu system that utilizations worldwide information.

In [6], NSGA-II is utilized to set the dozing plan for sensor organizes with a specific end goal to expand the scope accomplished while limiting the quantity of sensors utilized. For this situation, a disconnected and unified strategy that requirements worldwide information is utilized. This particular instance of sensor systems, where the objective territory is from the earlier known and some data can be assembled heretofore for ideal settings is good with a brought together improvement utilizing learning of the entire system. Luckily, numerous works in the writing utilize a decentralized approach when taking care of issues in specially appointed systems. This is the situation of BAOA [7], which utilizes subterranean insect settlement advancement calculations for limiting the aggregate vitality utilization. In spite of the fact that it runs on the web, it requires worldwide learning (area of all hubs). A decentralized and online hereditary calculation that utilizations conventional and developmental diversion hypothesis for self-spreading hubs in a territory utilizing just nearby information is proposed in [3]. For a broad overview on streamlining calculations taking care of issues in specially appointed systems, please allude to [4].

\section{REVIEW OF EXISTING WORK}

This segment surveys the primary existing work found in the logical writing that applies transformative calculations to multihop specially appointed systems. We isolate this area into various subsections every one covering the methodologies exhibited for each unique specially appointed worldview, for example, MANETs, VANETs, and DTNs. 


\subsection{MANETS}

In [4], creators arrange the fundamental research points in which developmental calculations have been utilized as topology administration, broadcasting calculations, directing conventions, bunching approaches, convention advancement, versatility models, narrow minded practices, security issues, and different applications. We take after a comparable scientific categorization yet for this situation we center around the fundamental research points of MANETs over the most recent two decades, for example, topology administration, broadcasting, and steering conventions.

\subsubsection{TOPOLOGY MANAGEMENT}

Topology administration issues or organization issues are centered on finding the ideal topology for proficiently accomplishing an objective execution of the system. In [7], the creators utilize a solitary target enhancement calculation to get the ideal positions and speed of various helper hubs in a railroad station situation. The goal is to expand the warning separation at which a prepare moving toward the station gets the data. In [8], the availability of team part acting a debacle situation is enhanced by the arrangement of static assistant guide hubs that are utilized as parcel forwarders. The streamlining issue is illuminated by applying a solitary target hereditary calculation. In [2], the availability of the system is estimated as the reach ability accomplished by communicating parcels sent by the group individuals. The creators utilize the notable hazardous situation portability demonstrate [8]in which the hazardous situation is isolated into various specialized territories. In [9], the creators utilize a PSO calculation to convey versatile hubs, called specialists, with a specific end goal to enhance the availability of the system.

\subsubsection{BROADCASTING ALGORITHMS}

Broadcasting is essential all-to all correspondence component generally utilized as a part of MANETs[9]. The primary target of a telecom calculation is to productively disperse a message all through the system. Notwithstanding its effortlessness, the outline of broadcasting calculations has drawn the consideration of the specially appointed group throughout the previous two decades [7][9]. The easiest telecom calculation called flooding has been exhibited ordinarily to be wasteful, causing the outstanding communicate storm problem[8].Many broadcasting calculations for the most part in light of topological parameters can be found in the writing [6][7], for example, the thickness of hubs, relative separation, and counter based systems. As of late, developmental calculations like hereditary calculations have been utilized to upgrade the plan of broadcasting calculations. In [3], the creators unravel the outstanding least vitality communicate NP-difficult issue utilizing a half breed transformative calculation. The thought decides the arrangement of forwarder hubs in a MANET that ensures most extreme scattering with the base vitality utilization. In [2], the creators utilize the generally utilized NSGA-II multiobjective algorithm[7] to streamline the plan of a telecom calculation in view of likeness/disparity coefficients.

\subsubsection{ROUTING PROTOCOLS}

Steering conventions are hugely utilized as a part of MANETs. They are so critical for the right execution of a dynamic conveyed arrange like a MANET. The essential goal of a directing convention is to locate the most appropriate correspondence course between a source and a goal 
hub. The plan of steering conventions has been extremely dynamic in the most recent decades, causing a high number of directing conventions in the logical writing to show up[9]. Besides, it has been shown that the design of a directing convention can affect prominently on the got comes about[6][7]. Transformative calculations have been generally utilized for the parameter setup of directing conventions. The thought is to locate the most ideal parameters for the execution of a directing convention. In [4], the creators test a few multiobjective advancement calculations to improve a straightforward steering convention that discovers courses between two hubs in the system. As per the outcomes in [4], MODE calculation is equipped for discovering arrangements nearer to the Pareto Front and, when all is said in done, focalizes quicker than the NSGA-II.

\section{OPEN CHALLENGES}

Transformative calculations have been connected to versatile multihop impromptu systems for the most recent decade. Notwithstanding, there are as yet numerous improvement issues in these mind boggling systems that can be fathomed utilizing a reasonable transformative calculation. New designs of developmental calculations are constantly proposed, for example, coevolutionary calculations and parallel transformative calculations. Since developmental calculations request high computational assets, the parallelization of the errands identified with the advancement strategy will permit executing hereditary calculations utilizing numerous processors and centers, decreasing the calculation time.

Hereditary writing computer programs are additionally a field that can be additionally researched in portable multihop specially appointed systems. Hereditary programming does likewise as hereditary calculations yet utilizing PC programs. With hereditary programming, it is hypothetically conceivable to locate the best communicating calculations or the best sending procedure. Be that as it may, hereditary programming has additionally a few hindrances, for example, the calculation time or the over the top development of its people. Another imperative normal open test is the utilization of totally appropriated execution of developmental calculations. The greater part of the examinations exhibited in this overview utilized incorporated usage. Notwithstanding, getting worldwide data in a portable multihop arrange is exorbitant as far as message trades. Moreover, the portability of hubs makes it an absolute necessity that the data be traded ceaselessly with a specific end goal to have refreshed data about the present condition of the system.

Moreover, dispersed usage of transformative calculation should lead with shortage of assets since versatile remote compact gadgets have for the most part bring down assets contrasted and workstations or PCs. The primary preferred standpoint of a disseminated execution is that the calculation is run on the web, so the hubs can adjust to the neighborhood changing states of the system. When all is said in done the execution of versatile remote multihop systems is directed by utilizing an arrangement of execution measurements that decide the nature of the calculations a work in progress utilizing a multiobjective streamlining calculation will enable the fashioners to have all the conceivable arrangements and to figure out which parameters influence the execution measurements initially (Pareto Front). Thus, in DTNs the most utilized execution measurements, for example, the hit rate, conveyance cost, and conveyance idleness, are additionally balanced.

\section{CONCLUSIONS}

This study paper has concentrated on a promising examination field, for example, the use of developmental calculations for enhancement issues in multihop impromptu systems. We have 
exhibited the fundamental highlights and limitations that ought to be mulled over for the utilization of developmental calculations in versatile multihop specially appointed systems. Moreover, we have assessed the primary works found in the examination writing, featuring the fundamental difficulties that require additionally inquire about. We trust that the utilization of developmental calculations in portable multihop impromptu system is still in a beginning period of research. Soon, the use of all the more intense and circulated transformative calculations will be conceivable on account of the addition of computational energy of implanted electronic gadgets.

\section{References}

[1] D.G.Reina et al., "A Survey on the Application of Evolutionary Algorithms for Mobile Multihop Ad Hoc Network Optimization Problems", International Journal of Distributed Sensor Networks, Vol.16, pp.1-13.(2016) DOI: $10.1155 / 2016 / 2082496$

[2] M. Conti and S. Giordano, "Mobile ad hoc networking: milestones, challenges, and new research directions," IEEE Communications Magazine, (2014), Vol. 52, No. 1, pp. 85-96.DOI: 10.1109/MCOM.2014.6710069

[3] M. Conti and S. Giordano, "Multihop ad hoc networking: the theory," IEEE Communications Magazine, (2007), Vol. 45, No. 4, pp. 78-86.DOI: 10.1109/MCOM.2007.343616

[4] M. Conti and S. Giordano, "Multihop ad hoc networking: the reality," IEEE Communications Magazine, (2007), Vol. 45, No. 4, pp. 88- 95.DOI: 10.1109/MCOM.2007.343617

[5] M. Conti, C. Boldrini, E. Kanhere, E. Pagani, P. M. Ruiz, and M. Younis, "From MANET to people-centric networking: milestones and open research challenges," Computer Communications, (2015), Vol. 71, pp. 121.DOI: 10.1016/j.comcom.2015.09.007

[6] B. Blywis, M. G“unes, F. Juraschek, O. Hahm, and N. Schmittberger, "Properties and topology of DES-tesbed (2nd extended revision)," Tech. Rep. TR-B-11-01, Telematic and Computer System, Freie Universit’at Berlin, Berlin, Germany, (2011).

[7] H. Pucha, S. M. Das, and Y.C.Hu, "The performance impact of traffic patterns on routing protocols in mobile ad hoc networks," Computer Networks, (2007), Vol. 51, No. 12, pp. 3595-3616.DOI: 10.1016/j.comnet.2007.02.009

[8] M. Abolhasan, T. Wysocki, and E. Dutkiewicz, "A review of routing protocols for mobile ad hoc networks," Ad Hoc Networks, (2004), Vol. 2, No. 1, pp. 1-22.

[9] A. Boukerche, B. Turgut, N. Aydin, M. Z. Ahmad, L. Boloni, and D. Turgut, "Routing protocols in ad hoc networks: a survey," Computer Networks, (2011), Vol. 55, No. 13, pp. 3032-3080. DOI: 10.1016/j.comnet.2011.05.010

[10] D. G. Reina, S. L. Toral, P. Johnson, and F. Barrero, "A survey on probabilistic broadcast schemes for wireless ad hoc networks,” Ad Hoc Networks, Vol. 25, pp. 263-292, (2015).DOI: 10.1016/j.adhoc.2014.10.001 
Behavior of Manets with Evolutionary Algorithms 